\title{
Life, Liquids and Cryo-Electron Microscopy
}

\author{
Jacques Dubochet, Heidelberg
}

(European Molecular Biology Laboratory - EMBL)

Life was created in water and water is still the most abundant constituent of biological matter. During the first 50 years of its existence, however, electron microscopy carefully removed all water from the specimens it observed. There is a simple reason for this sad situation: the column of an electron microscope is under vacuum, in which liquid water would immediately evaporate. The science and art of electron microscopy thus consists principally of preparing dry specimens without changing the native, hydrated structure too severely.

For many years, scientists have also tried to find a method of keeping the water in the electron microscope. This can be done by freezing the specimen, and this avenue has been explored with great vigour. The name of Humberto Fernandez-Moran (F-M, 1985) is inseparable from this effort. In $1975 \mathrm{~K}$. Taylor and R. Glaeser in Berkeley succeeded in showing that cryo-electron microscopy of hydrated biological specimens is not only possible but can also give good results (Taylor \& Glaeser, 1975). The trouble, however, was that water crystallizes upon cooling, and the resulting freezing damage is frequently devastating. This difficulty was already well recognized 50 years ago, when the Swissborn scientist Father B. Luyet suggested that the solution lay in cooling the aqueous sample sufficiently rapidly to bring its molecules to rest before water can crystallize - a process called "vitrification". During the next 40 years, Luyet and his remarkable colleagues tried to realize this goal, and in so doing created and developed modern cryo-biology with its well-known medical, commercial and ethical implications. In spite of all their efforts, however, they did not succeed in achieving vitrification except in highly concentrated aqueous solutions. As a result, it became generally accepted that vitrification was fundamentally impossible. It was therefore a big surprise when, at the beginning of the 80 's, Brüggeler and Mayer in Innsbruck ( $B$ \& $M, 1980$ ) and our group at the European Molecular Biology Laboratory in Heidelberg (Dubochet \& McDowall, 1981) showed that vitrification is possible and even easy. The whole trick behind this finding is that the cooling speed increases when the size of the sample decreases, and that the critical cooling speed is easily reached when the size of the sample is in the micrometer range. It is the good fortune of the electron microscopist that this is just the size of sample he is used to observing.

One lucky event rarely comes alone. After the discovery of vitrification, a range of methods soon emerged to enable direct electron microscopical observation of virtually any aqueous specimen. This is quick and easy when the

Fig. 1 - Cryo-electron micrograph of a vitrified thin film of oil microdroplets emulsified with detergent in water (Dubochet et al., 1984). In the light regions of the micrograph the microdroplets are closely packed in a single layer (enlarged in insert). There are two layers in the darker regions.

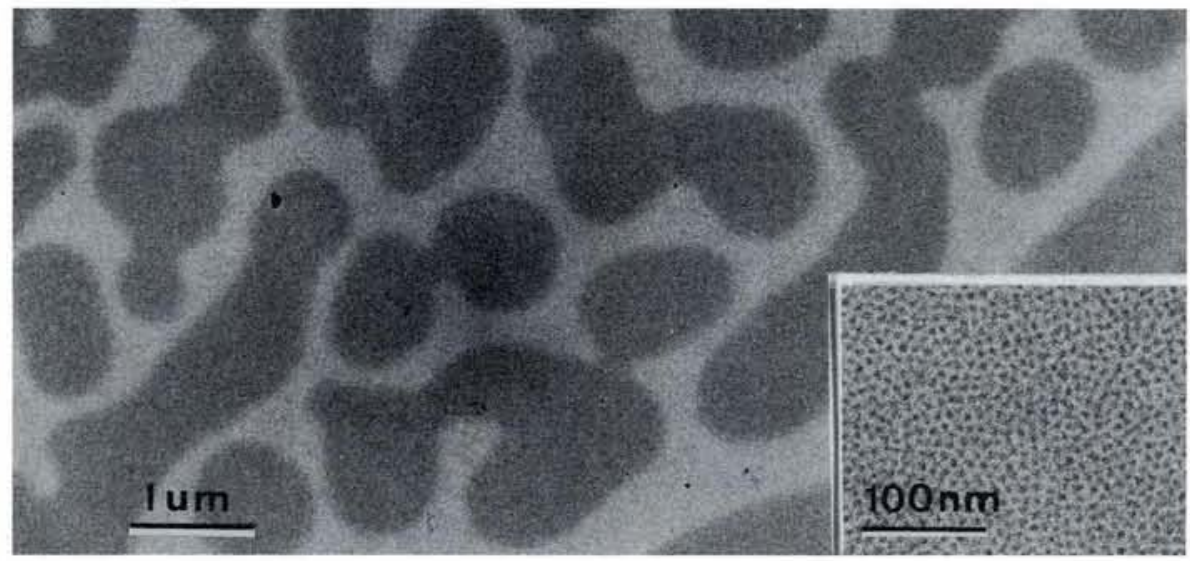

sample is an aqueous solution that can be cast in the form of a thin film (Adrian et al., 1984). It is more difficult and timeconsuming when bulky material or tissue has to observed, since the volume to be vitrified is larger and the sample must be cut into thin sections by cryo-ultramicrotomy below $-150^{\circ} \mathrm{C}$ (McDowall et al., 1983). Cryo-electron microscopy of vitrified samples is now used in a rapidly increasing number of laboratories and many structures previously studied by conventional methods are being reinvestigated in their aqueous environment. In general the results compare favourably with those obtained previously (Stewart \& Vigers, 1986).

Cryo-electron microscopy of vitrified samples is, however, more than just a method to overcome some of the artefacts of conventional electron microscopy. It allows the observation of a completely new class of structures, namely those formed by interactions taking place through the liquid medium. An example is shown in Fig. 1. It depicts a thin vitrified layer of a microemulsion (Dubochet et al., 1984). In this film, the ca $5 \mathrm{~nm}$ diameter microdroplets are packed in a non-crystalline layer (insert). There are, however, too many droplets for a single layer; some must be placed at a different level. They do not do this at random, but in a cooperative way thus forming regions where two densely packed layers are superimposed while the rest of the specimen contains only one layer. This alternation of one and two layers of microdroplets in the water film forms the structure seen in the figure. It is obviously quite ordered though it is neither periodic nor regular. It is, by the way, a challenging question to understand how this long range order is the consequence of a relatively disordered short range arrangement of the microdroplets.

The stucture shown in Fig. 1 is one example of a large class of structures which can be generally characterized by some remarkable properties:

a) The forces acting through the liquid water between the element of the structure (microdroplets in Fig. 1) are not strong enough to determine uniquely 
the position of one particle in respect to the other.

b) As a consequence, each particle is in a different microscopic environment and the structure is also macroscopically irregular.

c) As the particles are not tightly bound, thermal agitation is sufficient to displace them. The particles are thus mobile.

Altogether these properties can be described by the word "liquid".

Unfortunately, liquids have not been easy to study in the past. For example, $X$-ray diffraction, one of the most powerful tools for microstructural analysis, much prefers the particles to be arranged into a crystal. Variability between particles or local movement becomes systematically excluded from the recorded data. This is a great loss for biology and Henri Atlan expressed it in a picturesque way in the title of a book about life: "Between Crystal and Smoke" (1979). By this title he means that death has two structural forms: complete disorder, the smoke, or perfect order, the crystal. Life is this unstable state somewhere between the two extremes. From this point of view, electron microscopy is in a much better situation than any diffraction method. Looking for example at Fig. 2, or at most electron micrographs of biological samples, the large amount of order is generally the most conspicuous feature which strikes the observer. This order, however, is not simple. It is neither periodic nor regular and presents therefore some characteristics of a liquid structure. Nevertheless, conventional electron microscopy has its limitations and the need for dry specimens implies that all the weak bonds acting through the water are replaced by strong bonding of the dry particles.

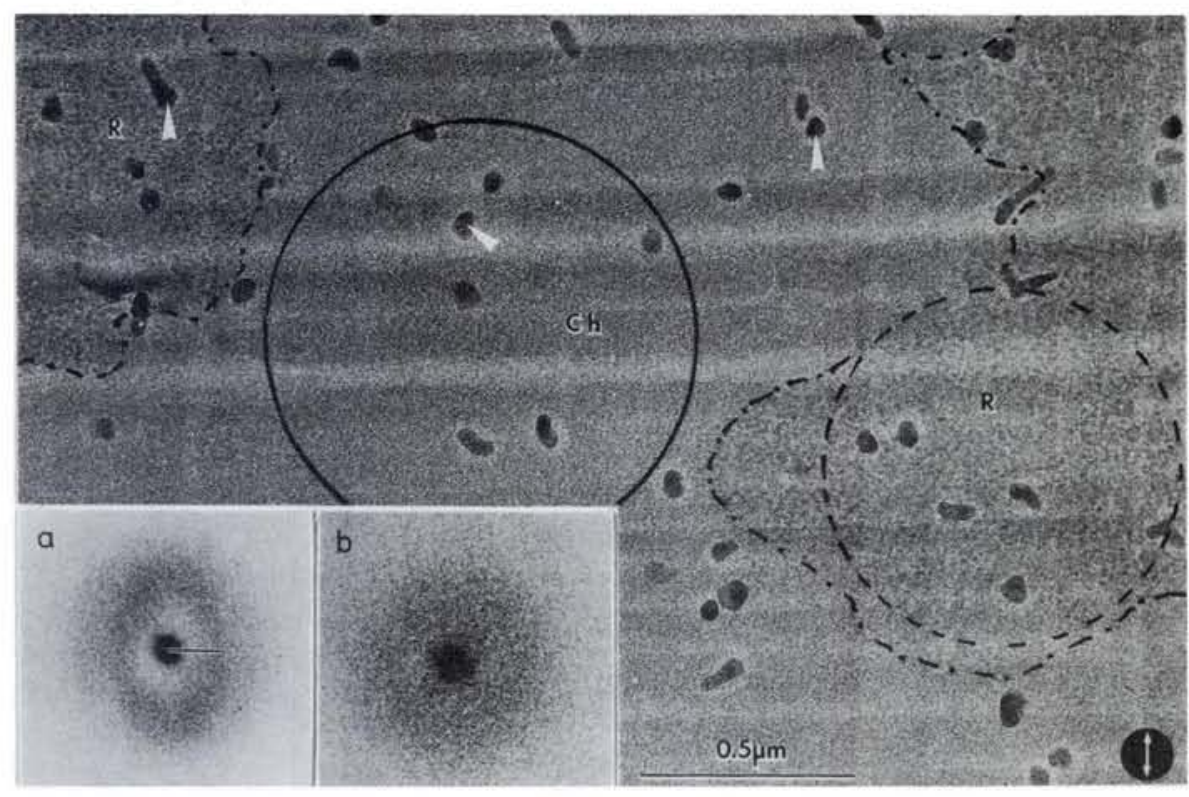

Fig. 3 - Thin vitrified section of a culture animal cell during mitosis. The unstained and unfixed sample was vitrified and cut by cryoultramicrotomy at $-160^{\circ} \mathrm{C}$ along the direction marked by a double arrow. A portion of a chromosome is visible in the delineated region (Ch). The rest of the micrograph represents the ribosome containing cytoplasm (R). White arrowheads point towards ice crystals contaminating the section surface. The optical diffractogram of the region circled in the chromosome is depicted in a) with a scale bar corresponding to $10 \mathrm{~nm}$. It shows a remarkable reflection around $11 \mathrm{~nm}$. It has the shape of an ellipse because the specimen was compressed during sectioning. The corresponding diffractogram of the circled region of the cytoplasm is shown in b). It corresponds essentially to a white spectrum (from McDowall et al., 1986; Fig. 6).

It was therefore a fascinating experience, when electron microscopy of vitrified specimens became a routine method, to observe that a multitude of specimens show a liquid structure. Even now, we are still surprised by the frequency and diversity of these observations. We came therefore to the idea that liquid structures are essential for the function of living organisms - an idea that can also be seen as trivial; the diversity of living organisms and the mobility of their structure are obvious daily

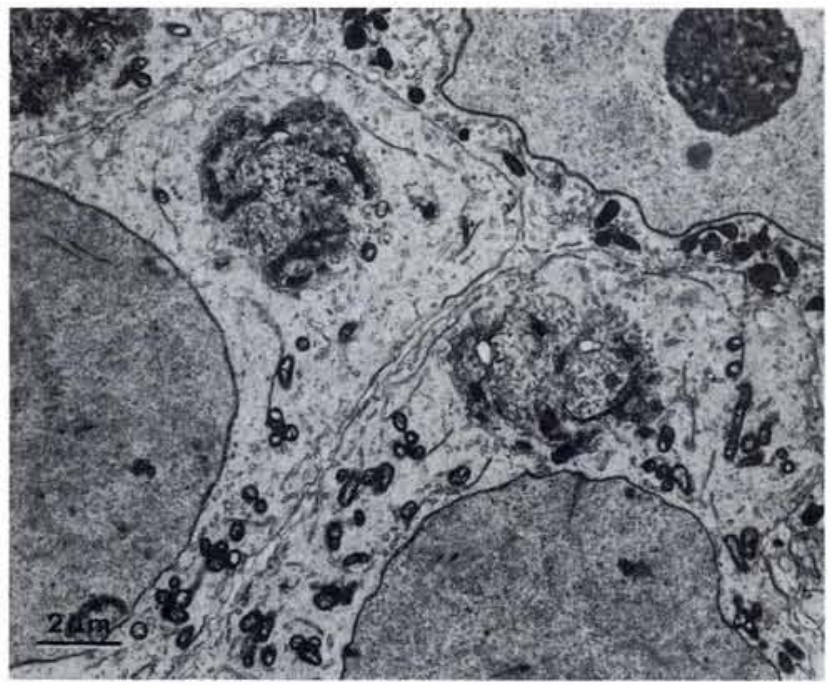

Fig. 2 - Seminiferous tubules of the rat testis. The sample was chemically fixed, dehydrated, stained and embedded in a plastic polymer before it was cut into a thin section and observed in a conventional electron microscope (courtesy G. Griffiths). observations. It is also not new; many scientists have worked a long way towards various "liquid hypotheses" (Frey-Wyssling, 1953). Cryo-electron microscopy of vitrified samples, however, provides for the first time a method for direct observation of liquid structures at the ultrastructural level. This possibility is illustrated in the two following examples.

The long DNA molecule forming the genetic material of a cell is condensed, together with many different proteins, into a mass called chromatin. It is generally accepted that the first step in the organization of chromatin is the nucleosome, a ca $10 \mathrm{~nm}$ particle formed by two loops of DNA around a protein core. Various models for the arrangement of nucleosomes have been proposed, leading to various hypothetical superstructures (Williams et al., 1986). Looking for these superstructures, we have observed mitotic chromosomes in intact vitrified cells (McDowall et al., 1986). The result depicted in Fig. 3 was surprising; it is a homogeneous granulation, characterized by a spacing of $c a 11 \mathrm{~nm}$, but with no detectable higher order arrangement. The simplest interpretation is that the nucleosomes, along the DNA fibre, are densely packed but in a disordered way.

The other example concerns a class of animal virus formed by a nuclear capsid containing the genetic material and sur- 
rounded by a membrane in which the surface spike proteins, necessary for recognition and fusion with the target cell, are incorporated. The nuclear capsid is synthesized in the cytoplasm of the infected cell, while the spike proteins are first incorporated into the cell outer membrane. The virus is formed during budding, namely when the nucleocapsid is coated by a portion of the cell membrane from which any protein other than virus spikes is excluded. The simplest hypothesis accounting for budding is that the spike proteins are selected by a one to one interaction with the proteins of the nucleocapsid template. If this is the case, the geometry of the envelope must be identical to that of the nucleocapsid. Cryo-electron microscopy could recently address this problem which has been intractable for conventional electron microscopy (Vogel et al., 1986; Fuller, submitted). The result of a three-dimensional reconstruction of the virus, is illustrated in Fig. 4. It shows that the icosahedral virus envelope has four morphological units per face. However, the nucleocapsid (not visible in the figure) which is also icosahedral, has only three units per face. No simple correspondence is therefore possible between capsid and membrane.

Chromatin and virus, presented in these two examples, are very different. They have, however, in common that their organization is not based on a strict complementarity. In the case of chromatin, the position of each nucleosome does not seem to be uniquely defined by the position of its neighbours. Chromatin seems therefore to have a liquid structure. This may throw a new light on the way it operates. In particular it suggests that rearrangement between nucleosomes or displacement of other molecules requires very little energy and that operations such as replication or transcription can be made locally without global rearrangement. In the case of the virus, the lack of complementarity between the nucleocapsid and the virus envelope shows that the first cannot be the only template for the second. Other interactions must be involved and it is simple to imagine that they take place between the spikes while they are floating in the cell membrane. Here also we have to deal with some form of a liquid.

From that point onwards a better description of the system becomes difficult. This is because we are not used to thinking in terms of non-crystalline order. There are perhaps cultural reasons for this; we like to see things equal, parallel and equidistant. Besides that, non-crys-

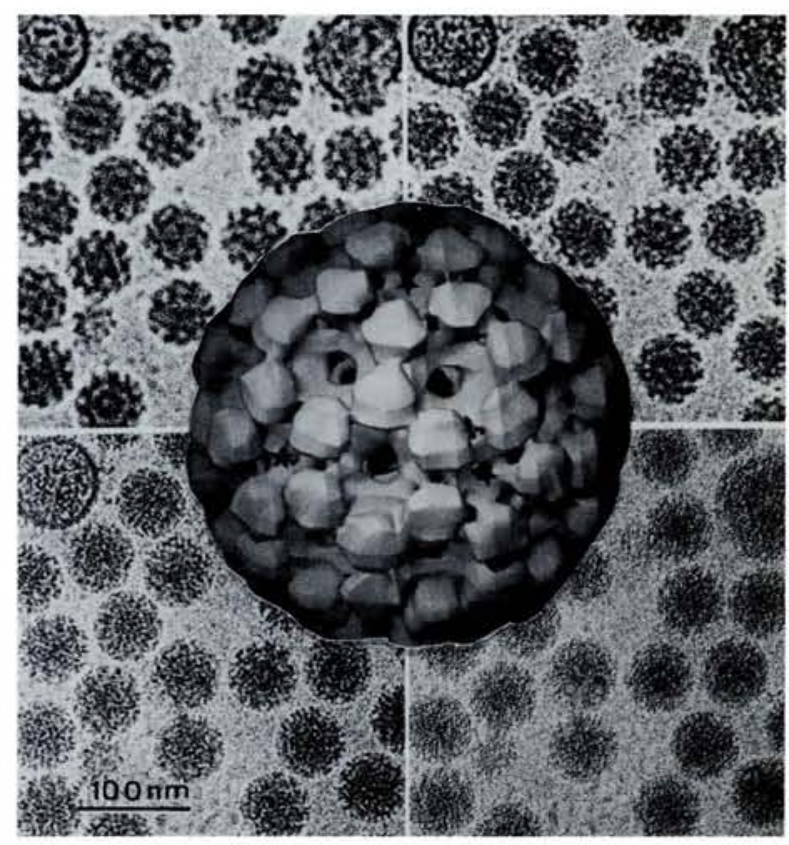

Fig. 4 - Semliki Forest Virus. Cryo-electron micrographs of a thin vitrified layer of native virus. Four micrographs were recorded at different focuses in order to retrieve all the information spectrum contained in the specimen. A three-dimensional model was calculated from these data. The surface of this model is shown in the center of the figure. It has icosahedral symmetry ( 3 adjacent subunits are marked with stars) and four morphological units per face (Vogel et al., 1986; Figs. 1 and 2, modified).

talline order is truly a difficult subject about which little is known. But things are changing fast. Our basic understanding of liquids is making rapid progress (Stillinger \& Weber, 1984); fractals (Mandelbrot, 1982) are causing a general spriteliness and the surprising discovery of paracrystals (Nelson, 1986) suggests how much more could be discovered in this field of science. I find it fascinating that it is also the case for biological structure and I suspect that cryo-electron microscopy of vitrified specimens will be a useful tool for exploring it.

\section{REFERENCES}

Adrian M., Dubochet J., Lepault J. and McDowall A.W., Cryo-electron Microscopy of Viruses, Nature 308 (1984) 32-36.

Atlan H. Entre le Cristal et la Fumée (Seuil, Paris) 1979.

Brüggeler P. and Mayer E., 'Complete Vitrification in Pure Liquid Water and Dilute Aqueous Solutions', Nature 288 (1980) 569-571.

Dubochet J. and McDowall A.W., 'Vitrification of Pure Water for Electron Microscopy', J. Microsc. 124 (1981) RP3-4.

Dubochet J., Adrian M., Teixeira J., Kadiyali R.K., Alba C.M., Mac Farlane D.R. and Angell C.A., 'Glass-forming Microemulsions: Vitrification of Simple Liquids and Electron Microscope Probing of Droplet Packing Modes', J. Physical Chem. 88 (1984) 6727-6732.

Fernandez-Moran H., ‘Cryo-electron Microscopy and Ultramicrotomy. Reminiscences and Reflections'. In: Advances in Electronics and Electron Physics, Supplement 16 (Academic Press, Inc.) 1985, pp. 167-223. Frey-Wyssling A., Submicroscopic Morphology of Protoplasm (Elsevier, Amsterdam) 1953.

Mandelbrot B.B., The Fractal Geometry of Nature (Freeman \& Co., San Francisco) 1982.

McDowall A.W., Chang J.-J., Freeman R., Lepault J., Walter C.A. and Dubochet J., 'Electron Microscopy on Frozen Hydrated Sections of Vitreous Ice and Vitrified Biological Samples'. J. Microsc. 131 (1983) 1-9. McDowall A.W., Smith J.M. and Dubochet J., 'Cryo-electron Microscopy of Vitrified Chromosomes in situ', EMBO J. 5 (1986) 1395-1402.

Nelson D.R., 'Quasicrystals', Sci. Amer. 255 (1986) 33-41.

Stewart M. and Vigers G., 'Electron Microscopy of Frozen-Hydrated Biological Material', Nature 319 (1986) 631-636.

Stillinger F.H. and Weber T.A., 'Packing Structures and Transitions in Liquids and Solids', Science 225 (1984) 983-989.

Taylor K.A. and Glaeser R.M., 'Electron Diffraction of Frozen, Hydrated Protein Crystals', Science 186 (1975) 1036-1037.

Vogel R.H., Provencher S.W., von Bonsdorff C.-H., Adrian M. and Dubochet J., 'Envelope Structure of Semliki Forest Virus Reconstructed from Cryo-electron Micrographs', Nature 320 (1986) 533-535.

Williams S.P., Athey B.D., Muglia L.J., Schnappe R.S., Gough A.H. and Langmore J.P., 'Chromatin Fibers are Left-handed Double Helices with Diameter and Mass per Unit Length that Depend on Linker Length', Biophys. J. 49 (1986) 233-248. 